\title{
Development of an X-ray tube for irradiation experiments using a field emission electron gun
}

Hidetoshi Kato ${ }^{\text {a, * }}$, Brian E. O'Rourke ${ }^{\text {a, }}$, Ryoichi Suzuki ${ }^{\text {a }}$, Jiayu Wang ${ }^{b}$, Takashi Ooi ${ }^{b}$ and Hidetoshi Nakajima ${ }^{b}$

${ }^{a}$ Research Institute for Measurement and Analytical Instrumentation (RIMA), National Metrology Institute of Japan (NMIJ), National Institute of Advanced Industrial Science and Technology (AIST), Tsukuba-Central 2, 1-1-1 Umezono, Tsukuba, Ibaraki 305-8568 Japan

${ }^{b}$ Pureron Japan Co.,Ltd., 1-37 Yoshima Industrial Park, Iwaki, Fukushima, 970-1144 Japan.

E-mail: katou-h@aist.go.jp

\begin{abstract}
A new X-ray tube using a ring-shaped emitter as a field emission electron source has been developed. By using a ring shaped cathode, $\mathrm{X}$-rays can be extracted along the axial direction through the central hole. This cylindrically symmetrical design allows for the tube to be arranged in the axial direction with the high voltage target at one end and the X-ray beam at the other. The newly developed X-ray tube can operate at a tube voltage of more than $100 \mathrm{kV}$ and at a tube current of more than $4 \mathrm{~mA}$, and can be used for irradiation experiments with an irradiation dose range from mGy up to kGy. The X-ray tube can be used immediately after turning on (i.e. there is no stand-by time). In the experimental model, we demonstrated stable electron emission at a tube voltage of $100 \mathrm{kV}$ and at a tube current of $4 \mathrm{~mA}$ during a 560 hour continuous test.
\end{abstract}

\section{Keywords}

X-ray source

Carbon nano-structures

Electron emission

Long lifetime 


\section{Introduction}

X-ray irradiation experiments are used in many research fields. Low-dose radiation effect in animals at doses from $1 \mathrm{mGy}$ to $10 \mathrm{~Gy}$ have been studied by many research groups [see ref 1 , and references therein], and such studies are used to investigate the relationship between received dose and cancer risk. X-ray irradiated doses of kGy order were used in the irradiation of food to control pathogenic bacteria [2], and the evaluation of radiation damage in semiconductor devices [3]. In conventional X-ray devices, typically the cathode and anode are aligned along the axis of a cylindrical X-ray tube. X-rays are extracted in a direction perpendicular to the axial electron beam. The anode is heated by collision with electrons and must be cooled, and either the anode or cathode must be floated to high-voltage. If the X-ray beam is to be extracted in the vertical direction, the device should be arranged in the horizontal direction. In such an arrangement the space footprint of the device is large. Compact high voltage $\mathrm{X}$-ray irradiation equipment is desirable because space is limited in the laboratory. Most X-ray tubes with a tube current of over $1 \mathrm{~mA}$ use a hot cathode type electron gun. Such hot cathodes have the disadvantage that some time is needed before they are operational, a stand-by time. Cold cathodes, on the other hand, need no warm up (stand-by) time and they can be formed in any shape required. X-ray tubes using a cold cathode can be used immediately. Various X-ray sources using carbon nanotubes (CNTs), carbon nanofibers or graphene flower cloth has been developed by many research groups [4-9]. Examples include compact X-ray sources [4, 5], a micro-focused X-ray source [6], a micro-CT scanner [7] for X-ray inspection, a multi-pixel X-ray array source [8] for micro-radiotherapy, and a stable X-ray source [9] for irradiation system. However, those previous X-ray sources typically operate at accelerating voltages of less than $50 \mathrm{kV}$ and lifetimes less than $100 \mathrm{~h}$. One notable exception to this generalization are the cold cathode, high-energy (tube voltage of more than $150 \mathrm{kV}$ ), pulsed X-ray sources for X-ray inspection that have been developed by Golden Engineering, Inc. [10].

At AIST we have been developing compact, light-weight, long-lifetime X-ray tubes for $\mathrm{X}$-ray imaging and X-ray fluorescence spectrometry using cold cathode electron emitters made from a particular type of chemical vapor deposition (CVD) grown carbon called Coniferous type Carbon Nano-Structure (CCNS) [11-13]. CCNS shows high stability at 
electron-current-densities of up to $100 \mathrm{~mA} / \mathrm{cm}^{2}$, which compares very favorably with CNTs [13], but it has proved difficult to make an emitter larger than around $16 \mathrm{~mm}$ in diameter. On the other hand, Pureron Co., Ltd have previously developed a different CVD based carbon emitter called Carbon Nano-structured Film (CNF) [14] as a field emission electron source grown to more than $40 \mathrm{~mm}$ in diameter on variously shaped emitter substrates. CNF can generate an electron-current-density of $20 \mathrm{~mA} / \mathrm{cm}^{2}$. In this paper, we report the development of an X-ray tube for irradiation experiments using a CNF emitter.

\section{Instrumentation}

A schematic diagram of the newly developed X-ray source X-ray tube for irradiation experiments is shown in Fig. 1. In the X-ray tube, all electrodes are cylindrically symmetrical, and X-rays are generated and extracted along the electron beam axis. The high voltage X-ray target and heat sink are arranged directly opposite the X-ray window. In this design the X-ray window is not influenced by the beam heating and the applied high-voltage on the X-ray target. The electron source is placed between the X-ray target and the X-ray window, and has an annular shape, with a central hole for X-rays to pass through. Emitted electrons from the emitter collide with the X-ray target generating X-rays. X-rays generated at the X-ray target pass through the hole in the center of the emitter and are transmitted through the X-ray window. The X-ray window and the emitter are kept at ground voltage. The potential of the X-ray target is set at positive high-voltage and determines the tube voltage. A grid is placed in front of the emitter surface. By adjusting the voltage on this grid the emission current from the emitter can be controlled, independent of the potential of the X-ray target. A focus lens between the grid and the X-ray target ensures the electron beam is adequately focused so that the X-ray spot size remains small. The focus lens is set at the same voltage as the grid.

The present cylindrically symmetrical design, in combination with extraction of the X-ray beam along the electron beam axis, allows several tubes to be arranged in close proximity in an array structure (see Fig.2), greatly increasing the available irradiation area while maintaining a high dose rate. Large area multi-pixel X-ray sources have been designed [15] and built [8] by several groups previously. These sources are designed with the aim of X-ray imaging or producing a large area X-ray beam with a well-defined spatial distribution. 
The electrodes in the experimental X-ray tube were designed with the aid of computer based simulations. The electric field and electron beam tracking were calculated by the finite-element analysis software Tricomp, version 8.0 (Field Precision, USA). A typical electron beam tracking result is shown in Fig. 3 where the distance between the emitter surface and the grid is fixed at $3 \mathrm{~mm}$. In the newly developed X-ray tube, the voltage of X-ray target must be higher than the grid voltage in order to focus the electron beam. In Fig. $3(\mathrm{a})$, the voltages of the emitter, the grid, the focus lens and the $\mathrm{X}$-ray target are set at $0 \mathrm{kV}$, $11 \mathrm{kV}, 11 \mathrm{kV}$ and $100 \mathrm{kV}$, respectively. The emitted electrons from the 40-mm-diameter emitter pass through the grid electrode, are focused to a beam by the focus lens, and are incident on the X-ray target. In all simulations, same focus lens was used, and deigned for focusing electron beam with no electron collision at focus lens and corresponding to wide tube voltage range of about $50 \mathrm{kV}$ to $160 \mathrm{kV}$. Fig. 3(a) shows the results of three simulations using the same applied voltages but varying the distance between the X-ray target and the grid. At distances of $51.5 \mathrm{~mm}, 36.5 \mathrm{~mm}$ and $26.5 \mathrm{~mm}$, the focused electron beam diameters are about $7 \mathrm{~mm}$, about $10 \mathrm{~mm}$ and about $14 \mathrm{~mm}$ (with an internal diameter of about $9 \mathrm{~mm}$ ), respectively. At the same applied voltage, the electron beam spot size is reduced when the distance between the X-ray target and the grid is increased. In Fig. 3 (b), the voltage of the grid and the focus lens is reduced from $11 \mathrm{kV}$ to $5 \mathrm{kV}$ causing a reduction in the electron beam spot size from 14-mm to $10-\mathrm{mm}$. In Fig. 3(c), the voltage of the X-ray target is increased from $44 \mathrm{kV}$ to $160 \mathrm{kV}$ causing a reduction in the electron beam spot size from 20-mm to 11-mm. Therefore, in the newly developed X-ray tube, the electron beam spot size is reduced at the larger separation between the X-ray target and the grid, low grid voltage and high X-ray target voltage.

Neglecting absorption in the air, the irradiation dose value is inversely proportional to the square of the distance between the X-ray target and irradiated sample. Therefore, for high dose, it is desirable that the distance between X-ray target and the grid (and therefore the window) is minimized. In the experimental model, the grid - target distance was fixed at $26.5 \mathrm{~mm}$ corresponding to the simulation shown in Fig 3(a). The X-ray tube voltage can be operated at more than about 4 times the grid voltage producing an electron beam with a spot size of about 20-mm-diameter (see Fig 3(c)), smaller than the 25-mm-diameter hole in the center of the emitter. 
Figure 4 shows a comparison of the electric field on the surface of the substrate for a flat surface and an uneven surface containing regularly spaced ridges. In this simulation the distance between the grid and substrate is $3 \mathrm{~mm}$, the applied voltage on the grid is $5 \mathrm{kV}$. Using the ridged surface the calculated electric field distribution also becomes uneven, with peak fields higher than the corresponding field for a flat surface. The peaks and small troughs in the electric field can be explained by the fact that the electric field is enhanced at the convex edges of the ridge more than in the central flat part. Therefore, we conclude that by using an emitter with a ridged surface, the applied voltage on the grid for a specific electron emission can be reduced and an electron energy at around the grid can be also reduced, leading to lower grid heating and reduced out-gassing from the grid by electron collisions. In the newly developed X-ray tube a ridged shaped CNF emitter was used.

The CNF emitter for the X-ray tube was grown on a stainless steel substrate using the DC plasma chemical vapor deposition (DCPCVD) method developed by Pureron Japan Co., Ltd. CNFs were grown on substrates in a mixed gas of $\mathrm{CH}_{4}$ and $\mathrm{H}_{2}$ (volumetric ratio of $\mathrm{CH}_{4}: \mathrm{H}_{2}=1: 25$ ) at a pressure of 50 torr for 60 min with a substrate temperature of around 1000 ${ }^{\circ} \mathrm{C}$. An electron microscopy image of the CNFs is shown in Fig. 5(a). The diameter of a single $\mathrm{CNF}$ ranges from several $\mu \mathrm{m}$ to $10 \mu \mathrm{m}$, and the CNFs grow randomly on the substrate. Photographs of the CNFs coated 40-mm-diameter substrate with 25-mm-diameter hole and a regular uneven structure are shown in Fig. 5(b).

The photograph of the constructed X-ray tube for irradiation experiments made by Pureron Japan Co., Ltd, and is shown in Fig. 6. The X-ray tube consists of a ceramic cylindrical outer tube with the $0 \mathrm{~V}$ side mounted on a standard ICF114 flange and the X-ray target connected to the other side. The ceramic tube also contains one intermediate ring where the grid and focus electrode potential is applied. In the X-ray tube, the electrodes consist of the CNFs emitter (see Fig. 5), the X-ray target (tungsten target brazed to a copper), the grid (molybdenum mesh, $\phi 0.035 \mathrm{~mm} \times 50$ wires/inch), and the focus lens. The $\mathrm{X}$-ray window is arranged at the distance of $75 \mathrm{~mm}$ from X-ray target surface. The X-ray target and CNFs emitter are insulated from the grid electrode by ceramics, respectively. An aluminum electrode with a smooth surface is mounted around the top of the X-ray target and acts to prevent electric discharge. Moreover, this electrode also increases the surface area of metal in contact with the target and helps to increase heat transfer to the surrounding oil. The 
applied voltage between the X-ray target and the grid can be increased up to maximum DC voltage of $+150 \mathrm{kV}$ by surrounding the tube in insulating oil. The X-ray tube voltage is determined by this applied voltage on the X-ray target. In order to prevent overheating of electrodes (mainly the X-ray target), the X-ray tube was cooled by circulating the oil through a chiller to maintain a temperature of $15^{\circ} \mathrm{C}$. The $\mathrm{X}$-ray tube was pumped by an ion pump and non-evaporable getter (SAES Getters St 172), and baked at a temperature of about $200{ }^{\circ} \mathrm{C}$ before use. The ultimate pressure in the X-ray tube is $3 \times 10^{-7} \mathrm{~Pa}$. During set-up operations of the X-ray tube, the emission current (i.e. the total of the grid current the tube current) from the CNFs emitter was increased gradually from $0 \mathrm{~mA}$ to $5 \mathrm{~mA}$. The fraction of electrons which passed through the grid was about $81 \%$.

\section{Results and discussion}

Plots of the emitted current against applied voltage (I-V) and the corresponding Fowler-Nordheim (F-N) plot of the CNFs emitter are shown in Figs. 7(a) and 7(b), respectively. The theory of field emission was proposed by R. H. Fowler and L. W. Nordheim [16] and typically the F-N equation is given by;

$$
\ln \left(I / E^{2}\right)=\operatorname{lr}\left(1.56 \times 10^{6} \times A \beta^{2} / \Phi\right)-\left(6.83 \times 10^{2} \times \phi^{1.2} / \beta\right) / E
$$

where $I$ is the emission current, $E$ is the electric field, $A$ is the effective emission area, $\beta$ is the field enhancement factor, and $\phi$ is the work function. A plot of $\ln \left(I / E^{2}\right)$ verses $1 / E$ will produce a straight line, the so called F-N plot. The present I-V plot was measured at an emission current (total of grid current and tube current) up to $5.5 \mathrm{~mA}$ as a function of the applied grid voltage, is shown in Fig. 7(a). For each data point in Fig 7(a), $V$ values are converted to $E$ values, and $\ln \left(I / E^{2}\right)$ are plotted against $1 / E$ to yield in Fig. 7(b). The linearity of F-N plot shows that electrons are emitted by field electron emission from the CNF. In Fig. 7(b), the values of the slope and intercept in the linear function of $1 / E$ were determined in a least-squares fit analysis of the experimental F-N plot. The result from this analysis, after being converted back into the I-V curve, is plotted as a solid line in Fig. 7(a). The values of the slope and intercept of the F-N plot are -49.88 and 1.59 , respectively. Considering that the CNF has the same tip shape as CNT, the field enhancement factor of CNF is calculated using the reported value for the work function for CNTs, i.e. $5 \mathrm{eV}$ [17]. The value of the field enhancement factor for the present CNF is calculated as 1531. This 
value is similar to the value of 1562 reported previously [13] for a CCNS emitter.

The measured emission current and applied voltage during long time continuous operation of the X-ray tube (at a tube current of $4.0 \mathrm{~mA}$ ) is shown in Fig. 8. The operation time was about 560 hours. The measured pressure in the X-ray tube is also shown. In this long time operation, the tube voltage and the grid current were fixed at $100 \mathrm{kV}$ and 0.93 $\mathrm{mA}$, respectively. The lines show the tube voltage of $100 \mathrm{kV}$, the tube current of $4.0 \mathrm{~mA}$, and the grid current of $0.93 \mathrm{~mA}$, respectively. The broken line shows the averaged grid voltage of $11.25 \mathrm{kV}$ in this long time operation. The measured grid voltages for the 560 hours are good agreement with the $11.25 \mathrm{kV}$ line. The fact that the grid voltage remained constant over the 560 hour operation shows that no significant degradation of the emitter occurred during this time, showing that the X-ray tube has potentially a long lifetime ( $>>$ 560 hours).

We measured absorbed dose rate of the X-ray tube at a tube voltage of $100 \mathrm{kV}$ and at a tube current of $4.0 \mathrm{~mA}$ by a Model FWT-92D Radiochromic Reader (Far West Technology, Inc.) dosimeter. In this experimental condition, a 36.6-mm-diameter and 2-mm-thick sapphire X-ray window was used which corresponds to an X-ray transmission rate equivalent to a 2-mm-thick sheet of aluminum. X-ray extraction angular range is about 27 degrees at the target. Geometrically, about $2.7 \%$ of the total X-ray emission passes through the central hole. The dimensions of the dosimeter were 1-cm-square and $44.5-\mu \mathrm{m}$-thick. The dosimeters were set at the positions of $1 \mathrm{~cm}$ and $15 \mathrm{~cm}$ from the X-ray window. The X-ray irradiation time was 333 hours. The absorbed dose rates were $200 \mathrm{~Gy} /$ hour and $26 \mathrm{~Gy} /$ hour at $1 \mathrm{~cm}$ and $15 \mathrm{~cm}$ from the X-ray window, respectively. These dose values are inversely proportional to a square of the distance $(8.5 \mathrm{~cm}$ and $22.5 \mathrm{~cm})$ from the X-ray production target surface. We compared the X-ray dose using an imaging plate (FujiFilm BAS-IP MS 2040) for the newly developed X-ray source and a conventional X-ray source with a 2-mm-thick aluminium filter, a target angle of 20 degree and an electron-X-ray angle of 90 degree in the same conditions (a tube voltage of $100 \mathrm{kV}$, a tube current of $0.05 \mathrm{~mA}$, an irradiation/exposure time of $1 \mathrm{~s}$, and a distance of $22.5 \mathrm{~cm}$ from X-ray target). The doses show almost the same value within the experimental error and the generated X-ray intensities are roughly uniform at all angles, consistent with a previous Monte Carlo simulation at $80 \mathrm{kV}[18]$. 
Furthermore, in order to check the X-ray energy distribution, we obtained X-ray radiographs of a stainless steel step wedge (stemless thickness of 1/16 - 6/16 inches) by the newly developed and a conventional X-ray source with an imaging plate, shown in Fig. 9. Both radiographs are obtained with the same processing. The darker part of the image corresponds to lower X-ray intensity. As the thickness of the stainless steel wedge increases only the higher energy end of the X-ray energy distribution can be transmitted. This result show the energy distribution is slightly shifted to lower energy for the newly developed source as compared to a conventional source. This shift to low energy is due to the fact that lower energy X-rays, generated by electrons with energies lower than the incident electrons after multiple scattering in the target, are produced with higher intensity in the backward direction than in the forward.

\section{Conclusion}

In the newly developed X-ray tube, all electrodes are cylindrically symmetrical, and generated X-rays are extracted along the beam axis. The high voltage X-ray target and heat sink are arranged directly opposite the X-ray window. In this design the X-ray window is not influenced by the beam heating and the applied high-voltage on the X-ray target. The electron source is placed between the X-ray target and the X-ray window, and has an annular shape, with a central hole for X-rays to pass through. Emitted electrons from the emitter collide with the X-ray target generating X-rays. X-rays generated at the X-ray target pass through the hole in the center of the emitter and are transmitted through the $\mathrm{X}$-ray window into the atmosphere. X-ray irradiation equipment using this $\mathrm{X}$-ray tube can have a very compact spatial footprint. The newly developed X-ray tube is operational with no warm-up time, unlike a conventional X-ray tube using a hot cathode. We demonstrated stable electron emission at a tube voltage of $100 \mathrm{kV}$ and at a tube current of $4 \mathrm{~mA}$ during a 560 hour continuous test. The expected lifetime of the X-ray tube is expected to be much longer than this test period. The dose rates of the newly developed X-ray tube is comparable to conventional X-ray tubes and depends on the tube voltage, tube current, and the distance from X-ray target. The present compact-sized X-ray tube can use for irradiation experiments with an irradiation dose range of from $\mathrm{mGy}$ up to $\mathrm{kGy}$ in the laboratory. Moreover, the present cylindrically symmetrical design, in combination with extraction of the X-ray beam along the electron beam axis, allows several tubes to be 
arranged in close proximity in an array structure, greatly increasing the available irradiation area.

\section{Acknowledgement}

The present work was supported by Revitalization Promotion Program (Matching Promotion) from Japan Science and Technology Agency (JST).

\section{References}

1) M. Tomita and M. Maeda, Mechanisms and biological importance of photon-induced bystander responses: do they have an impact on low-dose radiation responses, J. Radiat. Res. 56 (2015) 205.

2) B.S.M. Mahmoud, Inactivation effect of $X$-ray treatments on Cronobacter species (Enterobacter sakazakii) in tryptic soy broth, skim milk, low-fat milk and whole-fat milk, Lett. Appl. Microbiol. 49 (2009) 562.

3) T. Kudo, K. Kobayashi, S. Ono, T. Watanabe, H. Kinoshita, M. Okihara and T. Hatsui, Development of Experimental Methodology for Highly Efficient Wafer-Level Evaluation of X-Ray Radiation Effects on Semiconductor Devices, IEEE Trans. Nucl. Sci. 61 (2014) 1444.

4) J.-W. Jeong, J.-W. Kim, J.-T. Kang, S. Choi, S. Ahn, and Y.-H. Song, A vacuum-sealed compact x-ray tube based on focused carbon nanotube field-emission electrons, Nanotechnology 24 (2013) 085201.

5) Y. Iwai, K. Muramatsu, S. Tsuboi, A. Jyouzuka, T. Nakamura, Y. Onizuka, and H. Mimura, X-ray Tube Using a Graphene Flower Cloth Field Emission Cathode, Appl. Phys. Express 6 (2013) 105102.

6) K. Kawakita, K. Hata, H. Sato, and Y. Saito, Development of microfocused x-ray source by using carbon nanotube field emitter, J. Vac. Sci. Technol. B 24 (2006) 950.

7) G. Cao, Y. Z. Lee, R. Peng, Z. Liu, R. Rajaram, X. Calderon-Colon, L. An, P. Wang, T. Phan, S. Sultana, D. S. Lalush, J. P. Lu, and O. Zhou, A dynamic micro-CT scanner based on a carbon nanotube field emission X-ray source, Phys. Med. Biol. 54 (2009) 2323.

8) S. Wang, X. Calderon, R. Peng, E. C. Schreiber, O. Zhou, and S. Chang, A carbon nanotube field emission multipixel x-ray array source for microradiotherapy application, Appl. Phys. 
Lett. 98 (2011) 213701.

9) S. Kita, Y. Watanabe, A. Ogawa, K. Ogura, Y. Sakai, Y. Matsumoto, Y. Isokane, F. Okuyama, T. Nakazato, and T. Otsuka, Field-emission-type x-ray source using carbon-nanofibers, J. Appl. Phys. 103 (2008) 064505.

10) Golden Engineering, Inc. website: XR200 X-RAY SOURCE, http://www.goldenengineering.com/pdf/XR200OpNew.pdf (accessed September 2015).

11) R. Suzuki, Y. Kobayashi, and Y. Ishiguro, Cold Cathode X-Ray Source Using Coniferous Carbon Nano-Structure, Adv. X-Ray. Chem. Anal. Japan 41 (2010) 201.

12) H. Kato, B. E. O'Rourke, and R. Suzuki, Electron Gun using Coniferous Carbon Nano-Structure, JJAP Conf. Proc. 2 (2014) 011302.

13) H. Kato, B. E. O'Rourke, and R. Suzuki, Stable and high current density electron emission using coniferous carbon nano-structured emitter, Diamond \& Related Materials $55(2015) 41$.

14) J. Wang and T. Ito, High-current-density electron emissions from nano-carbon films fabricated by high-power microwave-plasma chemical vapour deposition, Diamond \& Related Materials 14 (2005) 1469.

15) E. J. Grant, C. M. Posada, C. H. Castano, H. K. Lee, A Monte Carlo simulation study of a flat-panel X-ray source, Appl. Radiat. Isotopes 70 (2012) 1658.

16) R. H. Fowler, and L. Nordheim, Electron Emission in Intense Electric Fields, Proc. Roy. Soc. A 119 (1928) 173.

17) M. Shiraishi and M. Ata, Work function of carbon nanotubes, Carbon 39 (2001) 1913.

18) M. R. Ay, M. Shahriari, S. Sarkar, M. Adib and H. Zaidi, Monte Carlo simulation of x-ray spectra in diagnostic radiology and mammography using MCNP4C, Phys. Med. Biol. 49 (2004) 4897. 


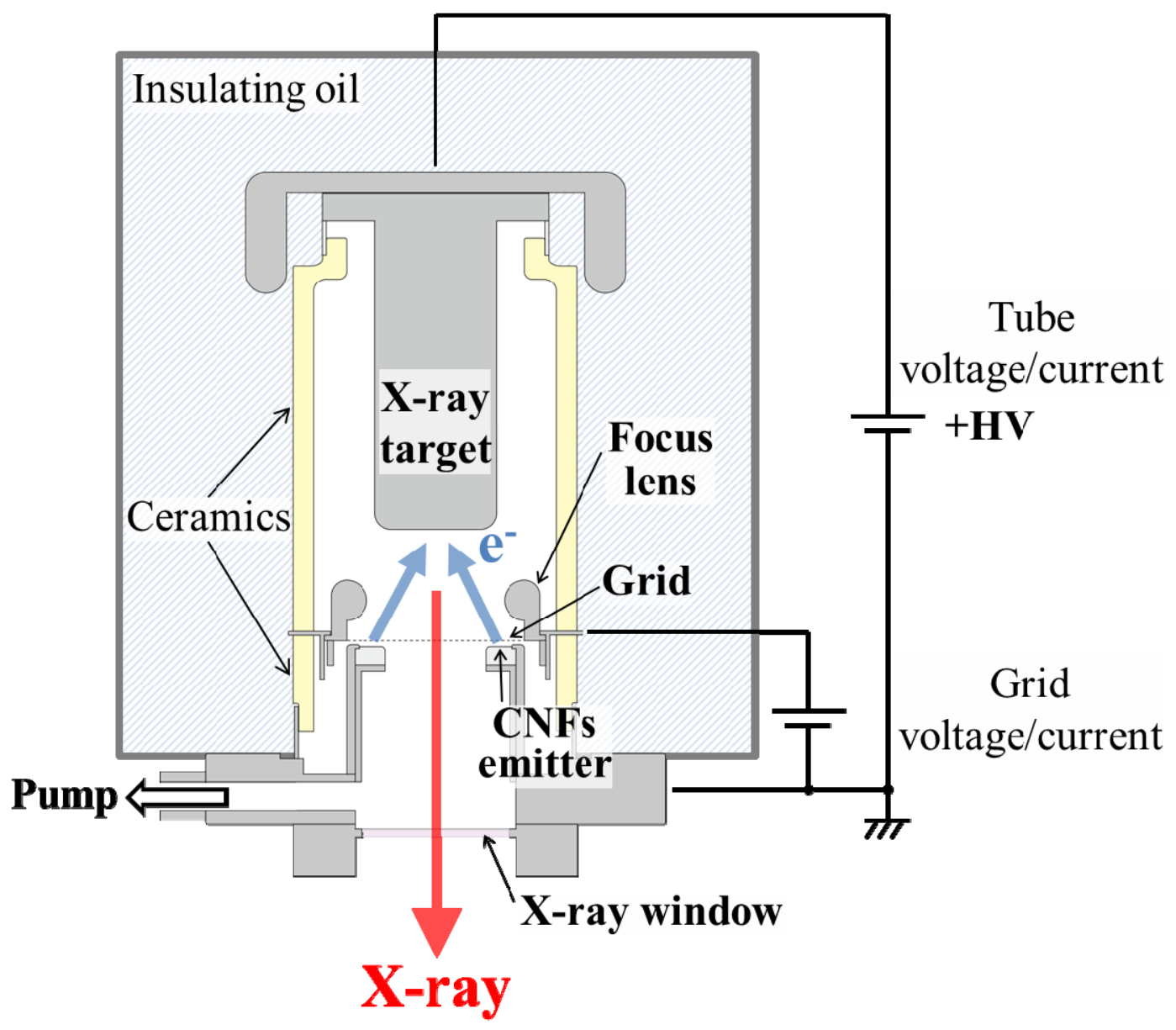

Fig. 1 Schematic diagram of newly developed X-ray tube for irradiation experiments. 


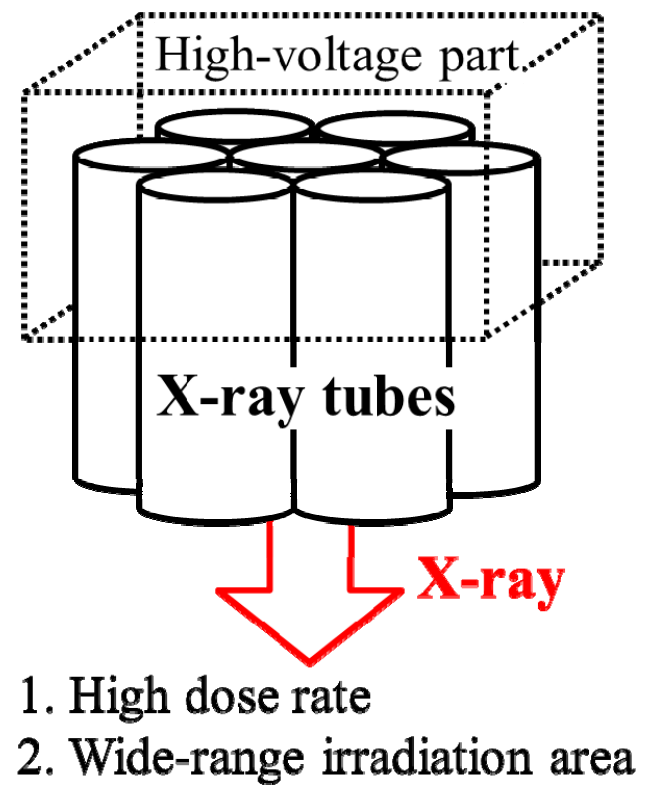

Fig. 2 The irradiation equipment for wide-range irradiation area and high dose rate using several present cylindrically X-ray tubes to be arranged in close proximity in an array structure. 

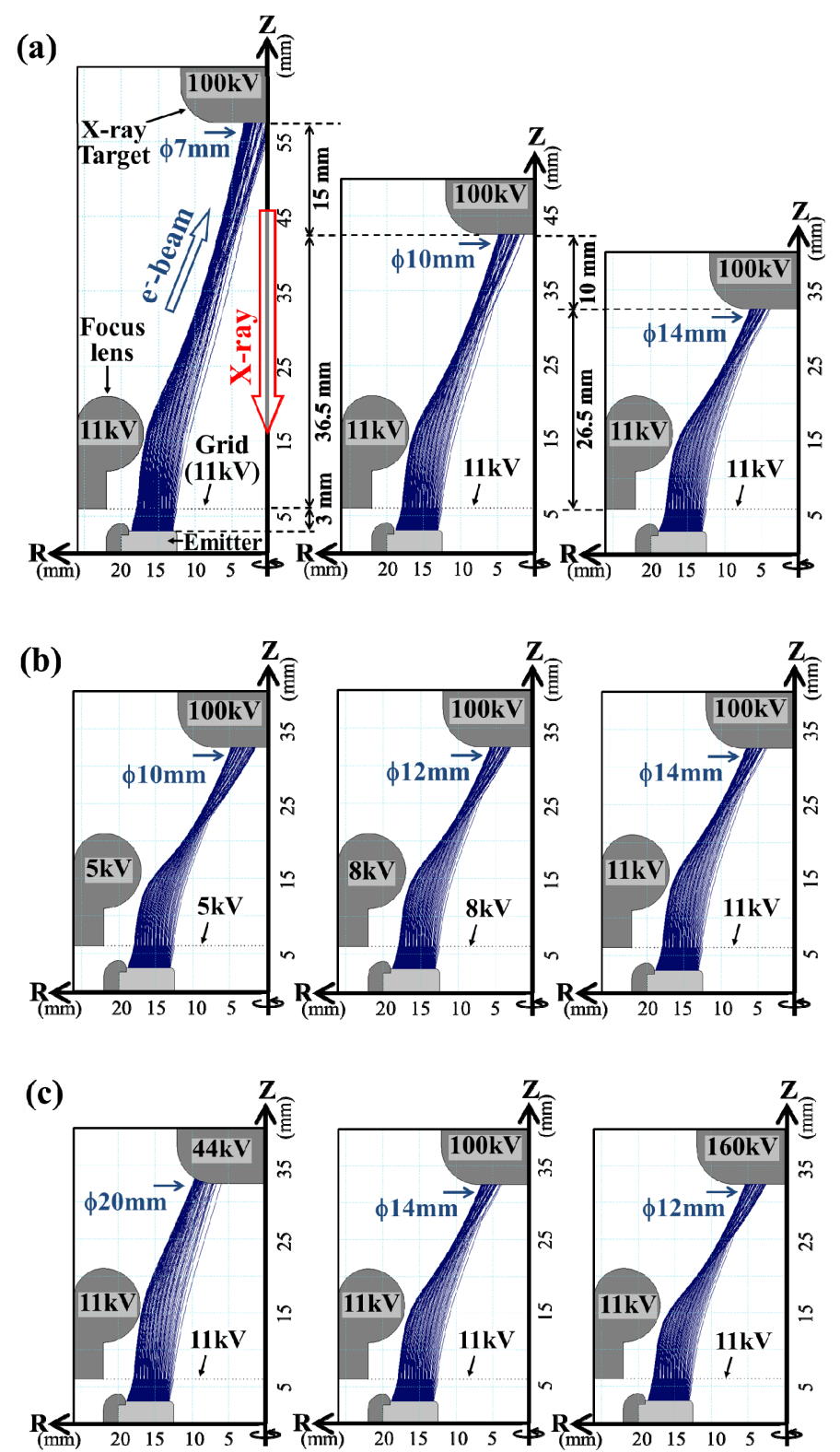

Fig. 3 Electron beam tracking simulations using the Tricomp simulation code (Field Precision) showing the variation of the electron beam spot size geometries and voltage configurations. (a) Moving the target closer to the electron gun increases the beam spot size at the same voltage conditions. (b) .Increasing the voltage on the focus electrode increases the beam spot size, (c) Increasing the voltage on the target reduces the beam spot size. 


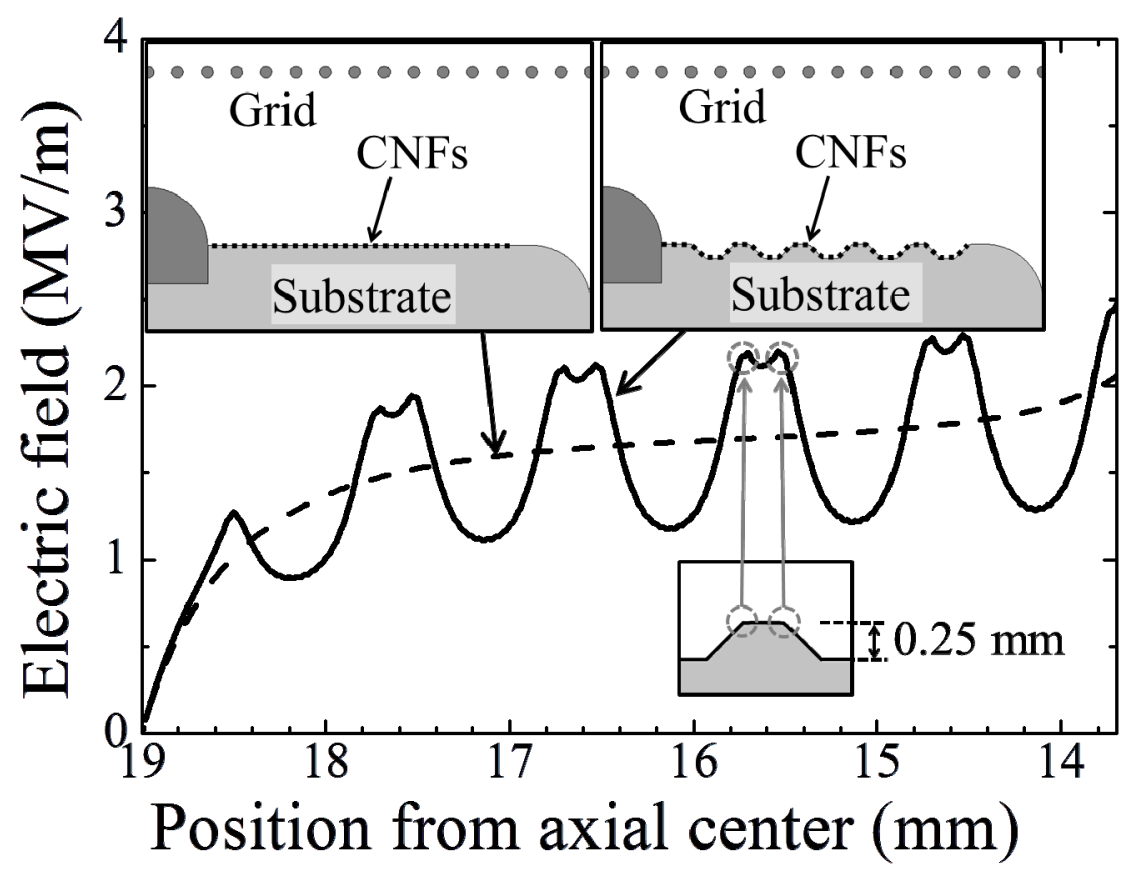

Fig. 4 The calculated electric field at the surface of a flat emitter (broken line) and an emitter with a ridged shaped surface (solid line). 


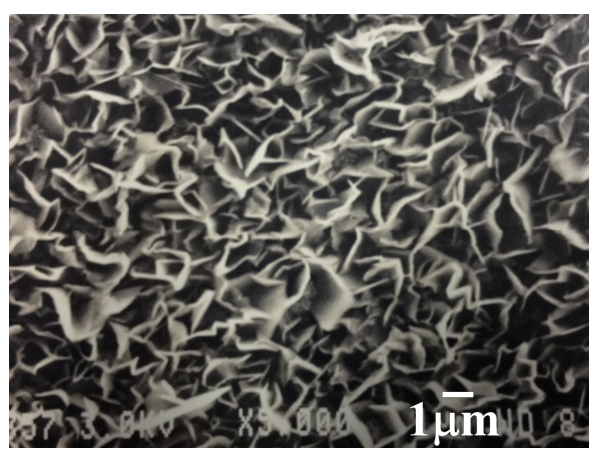

(a)

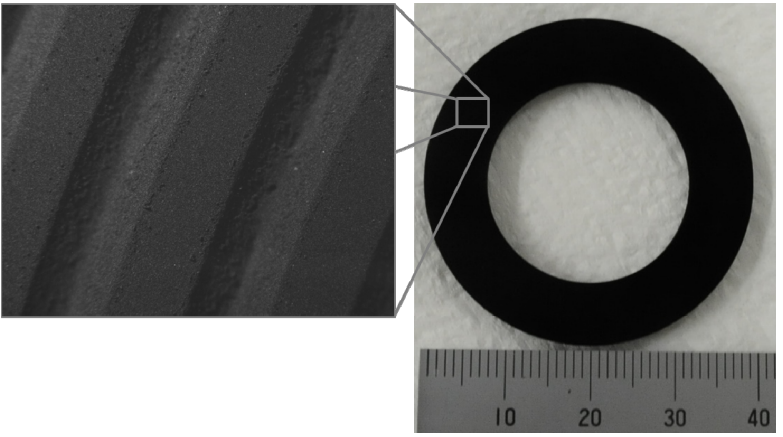

(b)

Fig. 5 (a) SEM image and (b) photographs of the upper-side view of the CNF on the substrate. 


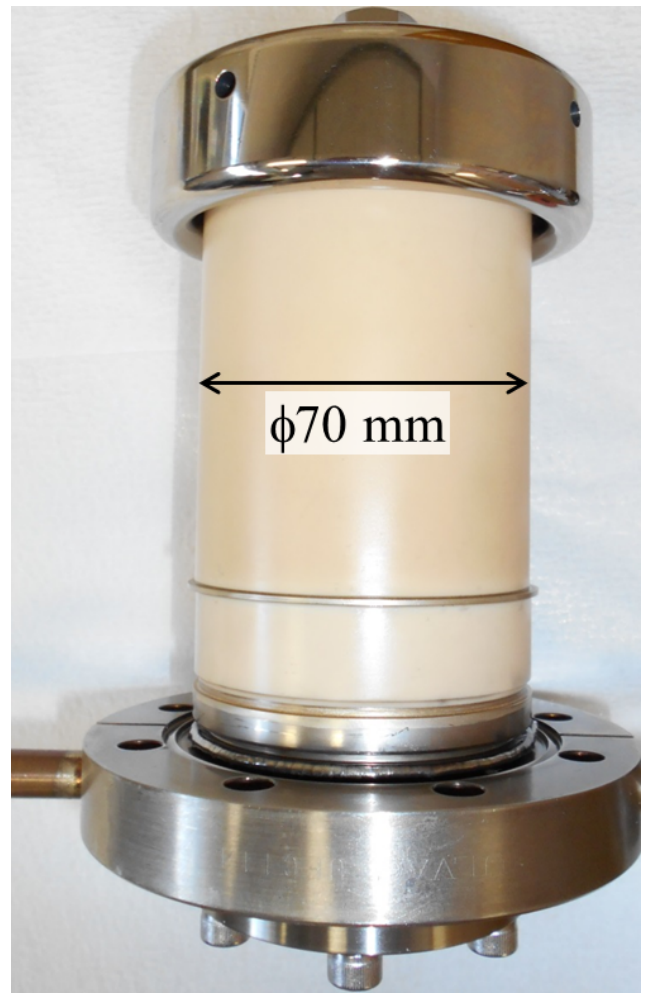

Fig. 6 Photograph of an experimental model X-ray tube for irradiation experiments. 

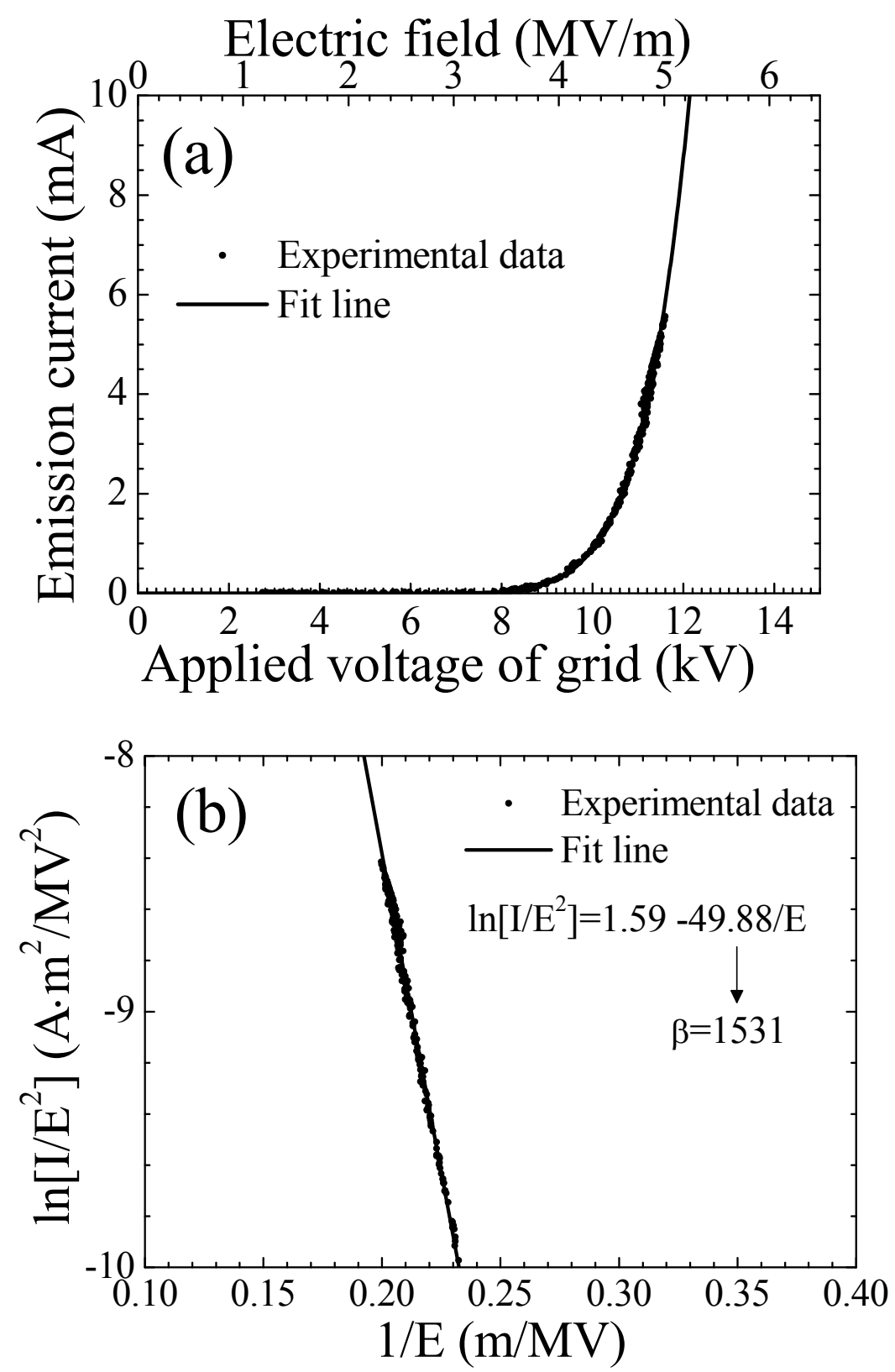

Fig. 7 (a) I-V characteristic and (b) Fowler-Nordheim (F-N) plot of the CNF emitter. The solid lines show the fit to the present data using Eq. (1). 


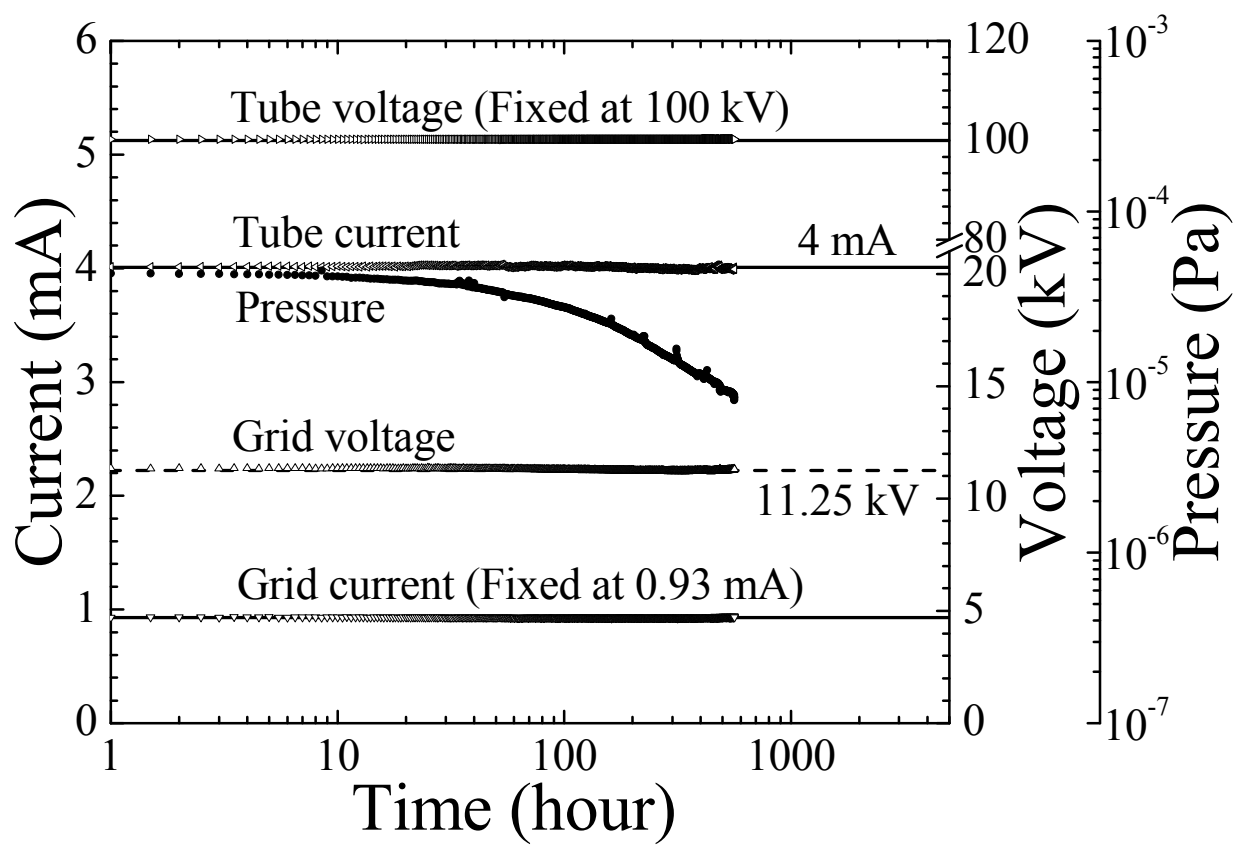

Fig. 8 Measured electron emission current and applied voltage during a long time continuous test at an X-ray tube current of $4.0 \mathrm{~mA}$. The measured data shows the tube voltage (right triangle), the tube current (left triangle), the grid voltage (up triangle), the grid current (down triangle) and pressure of X-ray tube (full plot). 


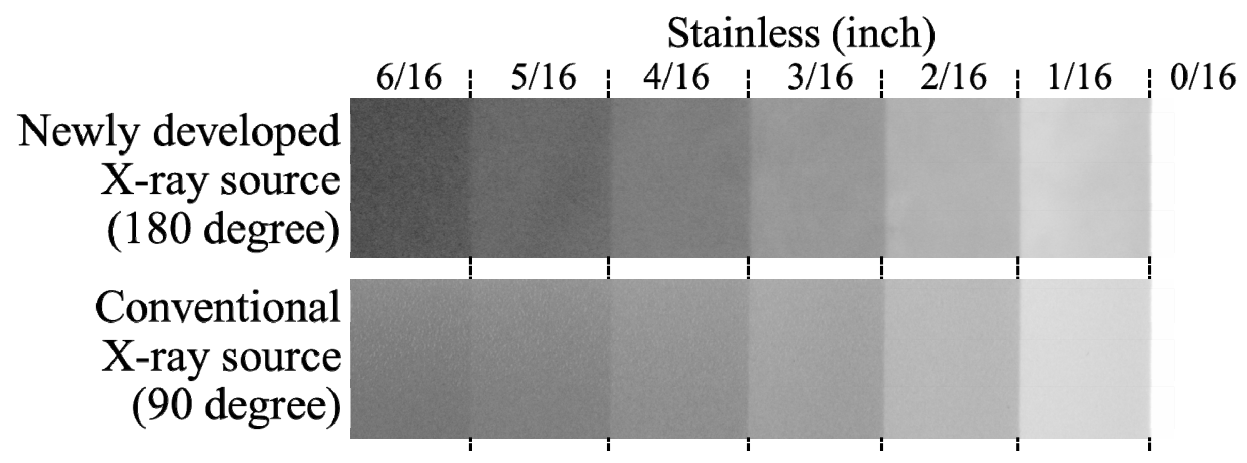

Fig. 9 X-ray radiographs of a step wedge obtained using the newly developed X-ray source (X-ray beam at 180 degrees to the electron beam) and a conventional X-ray source (X-ray beam at 90 degrees to the electron beam). 